\title{
NEURO-PSYCHOLOGY OF DEMENTIA AND OLD AGE: A COMPARATIVE STUDY OF MEMORY FUNCTIONING, VISUO-MOTOR PERCEPTION AND VISUO-SPATIAL CONSTRUCTIVE ABILITY
}

\author{
Soheli Datta [Assistant Professor and Clinical Psychologist] and \\ Padmini Patla [Psychologist], Department of Applied Psychology, \\ University of Calcutta, W.B., India
}

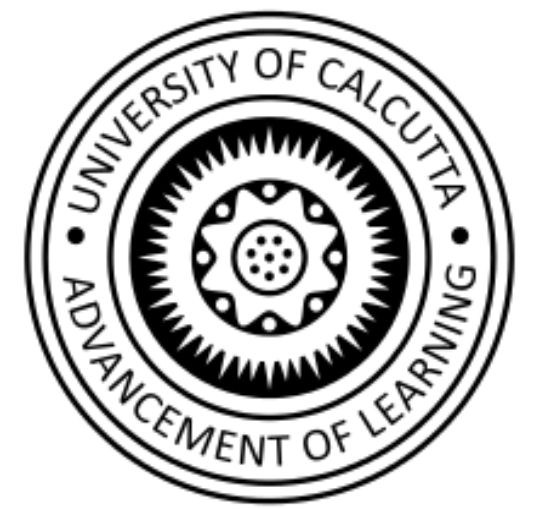

\begin{abstract}
Background: Dementia is characterized by symptoms manifested as weakened sensibility, understanding and will, with impaired recent memory, attention, reasoning and abstraction ability, severe enough to reduce a person's ability to perform everyday activities. Dementia can affect individuals in a number ofways, and progression of the disease depends upon the impact of the disease itself and the person's personality and state of health. Dementia might be primary or secondary to other disorders. In India, there is an overall prevalence rate of $0.84 \%$ for all dementias in the population aged 55 years and older and an overall prevalence rate of $1.36 \%$ among the 65 aged and older (Chandra et. al., 1998). In 2010, 3.7 million people aged over 60 years were estimated to have dementia (ARDSI, 2010).
\end{abstract}

\section{Method:}

Sample: The sample consisted of 60 individuals of age range 6075 years, males and females of dementia group $(\mathrm{N}=30)$ obtained from local government hospitals \& their normal counterparts $(\mathrm{N}=30)$. Normal control group was selected by ruling out presence of any psychopathology by using General Health Questionnaire 28 (Goldberg and Miller, 1979)

Tools Used: Case History Schedule, PGI Memory Scale (Pershad \& Wig, 1988, 1976), Bender Visual Motor Gestalt Test (Bender,1938), Clock Drawing Test (Shulman et al., 1986) and General Health Questionnaire (GHQ 28) (Goldberg and Miller, 1979).

Statistical Analysis: The result was analyzed using descriptive statistics and Independent samples t-test

\section{Result and Discussion:}

Results indicate significant difference between dementia and their normal counterpart on domains of memory functioning. These functions are tightly bound together in the first few years and support the ability to learn, communicate and plan for the future. Dementia is characterized by loss of neurons and synapses in the cerebral cortex and certain sub-cortical regions which results in group atrophy of the affected regions including degeneration of the frontal lobe, temporal and parietal lobe (Wenk, 2003). There is a significant difference between dementia and their normal counterpart on visuo-motor-perceptual organization and visuospatial constructive ability. This might be attributed to visuo-motor deficits in proximal and distal components of prolonged grasping movement times, inaccurate reaches to visual targets, difficulty in hand shaping for object size and inappropriate wrist orientation.

\begin{tabular}{|c|c|c|c|c|}
\hline Domains & Groups & Mean & SD & t-values \\
\hline Remote Memory & $\begin{array}{l}\text { Normal Control } \\
\text { Dementia }\end{array}$ & $\begin{array}{l}6.00 \\
3.13\end{array}$ & $\begin{array}{l}0.00 \\
1.30\end{array}$ & $12.022^{* \star}$ \\
\hline Recent Memory & $\begin{array}{l}\text { Normal Control } \\
\text { Dementia }\end{array}$ & $\begin{array}{l}4.90 \\
3.06\end{array}$ & $\begin{array}{l}0.30 \\
1.61\end{array}$ & $6.101^{* *}$ \\
\hline Mental Balance & $\begin{array}{l}\text { Normal Control } \\
\text { Dementia }\end{array}$ & $\begin{array}{l}7.80 \\
3.86\end{array}$ & $\begin{array}{l}1.58 \\
1.69\end{array}$ & $9.281^{* *}$ \\
\hline Attention and Concentration & $\begin{array}{l}\text { Normal Control } \\
\text { Dementia }\end{array}$ & $\begin{array}{l}8.80 \\
7.06\end{array}$ & $\begin{array}{l}1.06 \\
2.06\end{array}$ & $4.085^{\star *}$ \\
\hline Delayed Recall & $\begin{array}{l}\text { Normal Control } \\
\text { Dementia }\end{array}$ & $\begin{array}{l}8.43 \\
5.20\end{array}$ & $\begin{array}{l}1.67 \\
2.29\end{array}$ & $6.232^{* *}$ \\
\hline Immediate Recall & $\begin{array}{l}\text { Normal Control } \\
\text { Dementia }\end{array}$ & $\begin{array}{l}8.96 \\
4.20\end{array}$ & $\begin{array}{l}3.05 \\
2.34\end{array}$ & $6.782^{* *}$ \\
\hline Verbal Retention for similar pairs & $\begin{array}{l}\text { Normal Control } \\
\text { Dementia }\end{array}$ & $\begin{array}{l}4.53 \\
2.66\end{array}$ & $\begin{array}{l}0.77 \\
1.12\end{array}$ & $7.483^{\star *}$ \\
\hline $\begin{array}{l}\text { Verbal Retention for dissimilar } \\
\text { pairs }\end{array}$ & $\begin{array}{l}\text { Normal Control } \\
\text { Dementia }\end{array}$ & $\begin{array}{l}9.80 \\
4.73\end{array}$ & $\begin{array}{l}1.86 \\
2.01\end{array}$ & $10.106^{\star *}$ \\
\hline Visual Retention & $\begin{array}{l}\text { Normal Control } \\
\text { Dementia }\end{array}$ & $\begin{array}{l}11.16 \\
5.36\end{array}$ & $\begin{array}{l}1.85 \\
2.69\end{array}$ & $9.699^{\star *}$ \\
\hline Recognition & $\begin{array}{l}\text { Normal Control } \\
\text { Dementia }\end{array}$ & $\begin{array}{l}8.66 \\
5.00\end{array}$ & $\begin{array}{l}1.29 \\
2.13\end{array}$ & $8.046^{\star *}$ \\
\hline $\begin{array}{l}\text { Visuo-motor-perceptual } \\
\text { organization }\end{array}$ & $\begin{array}{l}\text { Normal Control } \\
\text { Dementia }\end{array}$ & $\begin{array}{l}2.06 \\
11.56\end{array}$ & $\begin{array}{l}2.74 \\
7.66\end{array}$ & $-6.390^{\star *}$ \\
\hline Visuo-spatial-constructive ability & $\begin{array}{l}\text { Normal Control } \\
\text { Dementia }\end{array}$ & $\begin{array}{l}4.46 \\
2.13\end{array}$ & $\begin{array}{l}0.86 \\
1.35\end{array}$ & $7.951^{\star \star}$ \\
\hline
\end{tabular}

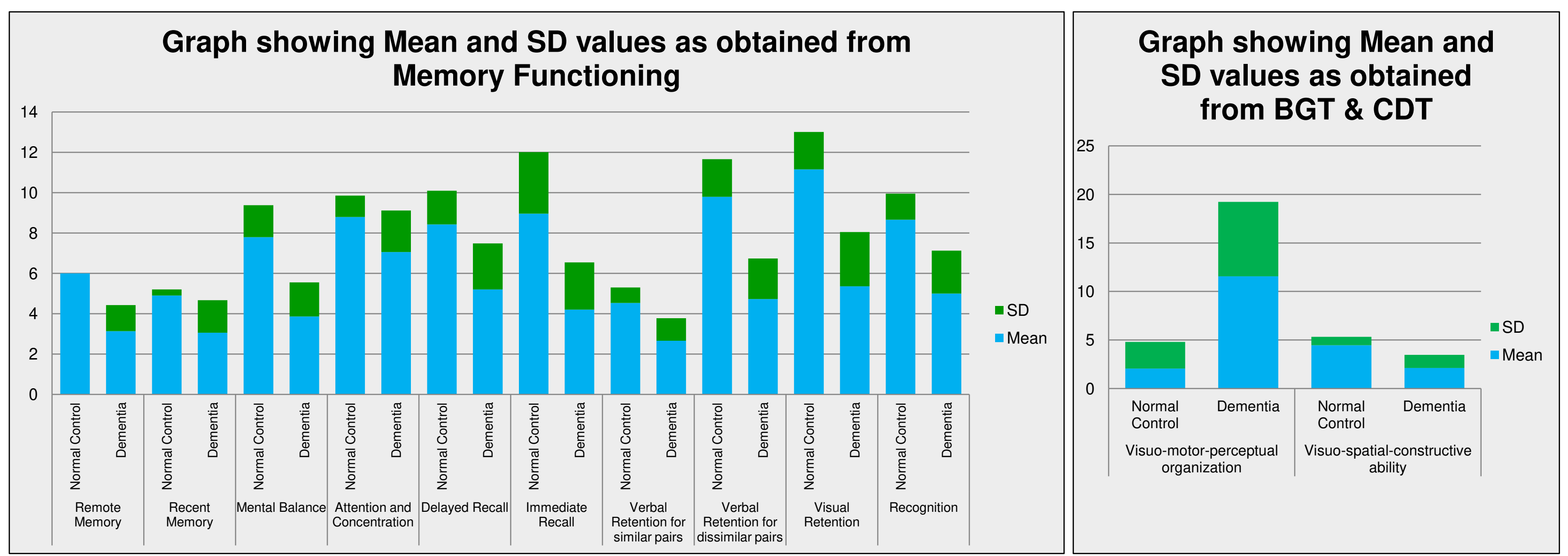

